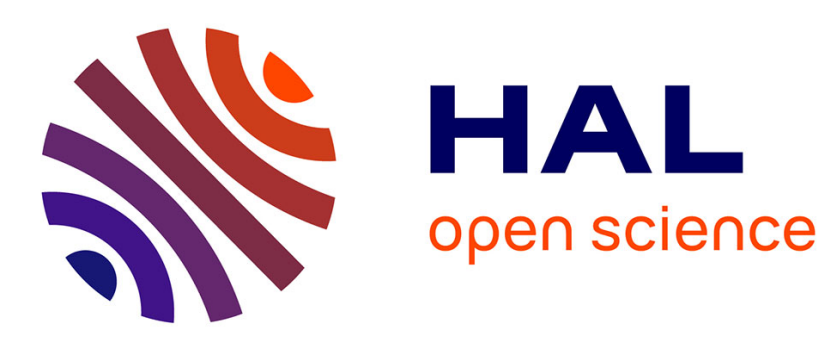

\title{
A Cartesian Scheme for Compressible Multimaterial Hyperelastic Models with Plasticity
}

\author{
Alexia de Brauer, Angelo Iollo, Thomas Milcent
}

\section{To cite this version:}

Alexia de Brauer, Angelo Iollo, Thomas Milcent. A Cartesian Scheme for Compressible Multimaterial Hyperelastic Models with Plasticity. Communications in Computational Physics, 2017, 22 (5), pp.1362-1384. 10.4208/cicp.OA-2017-0018 . hal-01586837

\section{HAL Id: hal-01586837 \\ https://inria.hal.science/hal-01586837}

Submitted on 13 Sep 2017

HAL is a multi-disciplinary open access archive for the deposit and dissemination of scientific research documents, whether they are published or not. The documents may come from teaching and research institutions in France or abroad, or from public or private research centers.
L'archive ouverte pluridisciplinaire HAL, est destinée au dépôt et à la diffusion de documents scientifiques de niveau recherche, publiés ou non, émanant des établissements d'enseignement et de recherche français ou étrangers, des laboratoires publics ou privés. 


\title{
A Cartesian Scheme for Compressible Multimaterial Hyperelastic Models with Plasticity
}

\author{
Alexia de Brauer, Angelo Iollo* \\ Univ. Bordeaux, IMB, UMR 5251, F-33400 Talence, France. CNRS, IMB, UMR \\ 5251, F-33400 Talence, France. INRIA, F-33400 Talence, France.
}

Thomas Milcent

Univ. Bordeaux, I2M, UMR 5295, F-33400 Talence, France. Arts et Métiers

Paristech, F-33607 Pessac, France.

\begin{abstract}
We describe a numerical model to simulate the non-linear elasto-plastic dynamics of compressible materials. The model is fully Eulerian and it is discretized on a fixed Cartesian mesh. The hyperelastic constitutive law considered is neohookean and the plasticity model is based on a multiplicative decomposition of the inverse deformation tensor. The model is thermodynamically consistent and it is shown to be stable in the sense that the norm of the deviatoric stress tensor beyond yield is non increasing. The multimaterial integration scheme is based on a simple numerical flux function that keeps the interfaces sharp. Numerical illustrations in one to three space dimensions of high-speed multimaterial impacts in air are presented.
\end{abstract}

Key words: compressible multimaterial, Eulerian elasticity, plasticity

* Corresponding author

Email addresses: alexia.de-brauer@math.u-bordeaux1.fr (Alexia de

Brauer), angelo.iollo@math.u-bordeaux1.fr (Angelo Iollo), thomas.milcent@u-bordeaux.fr (Thomas Milcent). 


\section{Introduction}

The numerical modelling of multimaterial rapid dynamics in extreme conditions is an important technological problem for industrial and scientific applications. Experiments are dangerous, need heavy infrastructures and hence are difficult and expensive to realize. The simulation of such phenomena is challenging because they couple large deformations and displacements in solids to strongly non-linear behaviour in fluids. In what follows, we privilege a fully Eulerian approach based on conservation laws, where the different materials are characterized by their specific constitutive laws. This approach was introduced in [10] and subsequently pursued and extended for example in [19], [9], [2], [13]. For specific applications, a Lagrangian approach like in [17] or [15] can be more suitable.

In this work we extend to elasto-plastic flows the schemes presented in $[11,5]$ for hyperelastic multimaterials. This scheme is based on a simple modification of the numerical flux function at the multimaterial interface that allows an efficient code parallelization. Thanks to this scheme, there is no need of defining a ghost fluid through the material boundaries to avoid accuracy and stability issues. In addition, this interface remains sharp.

Plasticity modelling is an open issue. The models are phenomenological and several problematic points still need further investigation, see for example [20]. Here we follow an established literature [16,18,12,1,22,6,21] where the deformation is viewed as the composition of a purely elastic and a purely plastic mapping. This approach has the advantage that plastic effects are modeled as a source term in the equation for the elastic deformation tensor. Also, using appropriate constitutive equations for the plastic phenomenon, it is possible to respect thermodynamic consistency (entropy is increasing) and phenomenological constraints as volume invariance of the plastic flow. In particular, we show here that with the plasticity model adopted in [18], the deviatoric stress tensor norm is actually non increasing during the plastic process for the neohooken hyperelestic model.

The applications we present are illustrations of the stiff phenomena occurring when high speed projectiles impact on shields. These examples include 
a one-dimensional case where we can compare the numerical results to an exact solution, a two-dimensional impact on a plastic layer, two and threedimensional impacts on framed plastic shields.

\section{Eulerian hyperelastic model}

This model was already discussed in $[10,23,24,19,3,8]$. We follow here the formulation presented in [5] and extend it to plasticity modelling. The equations of mass, momentum, deformation and energy conservation are given by

$$
\left\{\begin{array}{r}
\partial_{t} \rho+\operatorname{div}_{x}(\rho u)=0 \\
\partial_{t}(\rho u)+\operatorname{div}_{x}(\rho u \otimes u-\sigma)=0 \\
\partial_{t}\left(\nabla_{x} Y\right)+\nabla_{x}\left(u \cdot \nabla_{x} Y\right)=0 \\
\partial_{t}(\rho e)+\operatorname{div}_{x}\left(\rho e u-\sigma^{T} u\right)=0
\end{array}\right.
$$

The physical variables are the density $\rho(x, t)$, the velocity $u(x, t)$, the total energy per unit mass $e(x, t)$ and the Cauchy stress tensor in the physical domain $\sigma(x, t)$. Here $Y(x, t)$ is the backward characteristics that for a time $t$ and a point $x$ in the deformed configuration, gives the corresponding initial point in the initial configuration. The equation on $\nabla_{x} Y$ is required in order to record the deformation in the Eulerian frame. The initial density $\rho(x, 0)$, the initial velocity $u(x, 0)$, the initial total energy $e(x, 0)$ and $\nabla_{x} Y(x, 0)=I$ are given together with appropriate boundary conditions.

We assume that the internal energy per unit mass $\varepsilon=e-\frac{1}{2}|u|^{2}$ is the sum of a term accounting for volumetric deformation depending on the density $\rho$ and the entropy $s$, and a term accounting for isochoric deformation depending on the modified left Cauchy-Green tensor $\bar{B}$ given by

$$
\bar{B}(x, t)=\left[\nabla_{x} Y\right]^{-1}\left[\nabla_{x} Y\right]^{-T} / J^{\frac{2}{3}}(x, t) \quad J(x, t)=\operatorname{det}\left(\left[\nabla_{x} Y\right]\right)^{-1}
$$

We choose a general constitutive law that models gas, fluids and elastic solids given by 


$$
\varepsilon\left(\rho, s, \nabla_{x} Y\right)=\overbrace{\underbrace{\frac{\kappa(s) \rho^{\gamma-1}}{\gamma-1}+\frac{p_{\infty}}{\rho}}_{\text {stiffened gas }}+\frac{\chi}{\rho_{0}}(\operatorname{Tr}(\bar{B})-3)}^{\text {neohookean elastic solid }}
$$

We obtain according to the formula $\sigma=\rho \frac{\partial \varepsilon}{\partial F} F^{T}$ where $F=\left[\nabla_{x} Y\right]^{-1}$ is the deformation tensor

$$
\sigma\left(\rho, s, \nabla_{x} Y\right)=-p(\rho, s) I+2 \chi J^{-1}\left(\bar{B}-\frac{\operatorname{Tr}(\bar{B})}{3} I\right)
$$

where

$$
p(\rho, s)=\kappa(s) \rho^{\gamma}-p_{\infty}
$$

Here $\kappa(s)=\exp \left(\frac{s}{c_{v}}\right)$ and $c_{v}, \gamma, p_{\infty}, \chi$ are positive constants that characterize a given material. Parameter $p_{\infty}$ accounts for fluid or solid materials where intermolecular forces are present (see for example $[9,8]$ ). The last term in the energy expression models a neohookean elastic solid where the constant $\chi$ is the shear elastic modulus. Here we stick to a neohookean model as it has been shown to result in an hyperbolic system [5]. In order to be self contained we provide below the analytical wave speeds for the neohookean law.

Let us consider the one-dimensional problem in the $x_{1}$ direction $\Psi_{t}+(F(\Psi))_{, 1}=$ 0 associated to system (1). The wave speeds are the eigenvalues of $F^{\prime}(\Psi)$ and are given by

$$
\Lambda^{E}=\left\{u_{1}, u_{1}, u_{1} \pm \sqrt{\frac{\alpha_{1}}{\rho}}, u_{1} \pm \sqrt{\frac{\alpha_{2}}{\rho}}, u_{1} \pm \sqrt{\frac{\alpha_{3}}{\rho}}\right\}
$$

Here, $\alpha_{1}, \alpha_{2}$ and $\alpha_{3}$ are the roots of the polynomial of third order $X^{3}+$ $\operatorname{Tr}(\Sigma) X^{2}+\operatorname{Tr}(\operatorname{Cof}((\Sigma)) X+\operatorname{Det}(\Sigma)=0$ and 


$$
\Sigma=[\nabla \sigma][\nabla Y]:=\left(\begin{array}{ccc}
\sigma_{, 1}^{11} & \sigma_{, 2}^{11} & \sigma_{, 3}^{11} \\
\sigma_{, 1}^{21} & \sigma_{, 2}^{21} & \sigma_{, 3}^{21} \\
\sigma_{, 1}^{31} & \sigma_{, 2}^{31} & \sigma_{, 3}^{31}
\end{array}\right)\left(\begin{array}{ccc}
Y_{, 1}^{1} & Y_{, 2}^{1} & Y_{, 3}^{1} \\
Y_{, 1}^{2} & Y_{, 2}^{2} & Y_{, 3}^{2} \\
Y_{, 1}^{3} & Y_{, 2}^{3} & Y_{, 3}^{3}
\end{array}\right)
$$

where $\sigma_{, 1}^{i j}, \sigma_{, 2}^{i j}$ and $\sigma_{, 3}^{i j}$ denote the derivative of $\sigma^{i j}$ with respect to $Y_{, 1}^{1}, Y_{, 1}^{2}$ and $Y_{, 1}^{3}$ respectively. For the neohookean law we can show that

$$
\Sigma=\frac{2}{9} \chi J^{1 / 3}\left(\begin{array}{ccc}
-2 A_{11}-5 A_{22}-5 A_{33} & 6 A_{12} & 6 A_{13} \\
6 A_{12} & -9 A_{11} & 0 \\
6 A_{13} & 0 & -9 A_{11}
\end{array}\right)-\rho c^{2}\left(\begin{array}{ccc}
1 & 0 & 0 \\
0 & 0 & 0 \\
0 & 0 & 0
\end{array}\right)
$$

where $c^{2}=\left.\frac{\partial p}{\partial \rho}\right|_{s}$ and $A=J^{-\frac{4}{3}} \bar{B}$. We have proved that $\alpha_{i}>0$ for all deformations. Note that the initial expression of $\Sigma$ given in [5] depends on $\beta_{2}$ and $\beta_{3}$ but they actually vanish.

\section{$3 \quad$ Plasticity modelling}

Plasticity describes the deformation of a material undergoing non-reversible changes of shape in response to applied forces. When the force is unloaded the solid does not come back to the initial configuration. Experimentally plasticity occurs when the stress exceeds a critical value. Let the deviatoric part of the stress tensor

$$
\operatorname{dev}(\sigma)=\sigma-\frac{\operatorname{Tr}(\sigma)}{3} I
$$

We define the yield function of von Mises

$$
f_{V M}(\sigma)=|\operatorname{dev}(\sigma)|^{2}-\frac{2}{3}\left(\sigma_{y}\right)^{2}
$$


The surface $f_{V M}(\sigma)=0$ represents the yield surface and $\sigma_{y}$ is the plastic yield limit. We have

$$
\left\{\begin{array}{lll}
\text { if } \quad f_{V M}(\sigma) \leq 0 & \Longrightarrow & \text { elastic regime } \\
\text { if } \quad f_{V M}(\sigma)>0 & \Longrightarrow & \text { plastic regime }
\end{array}\right.
$$

In this paper, we restrict ourselves to the case of perfect plasticity where $\sigma_{y}$ is a constant. We have that

$$
|\operatorname{dev}(\sigma)|^{2}=\frac{1}{3}\left(\left(\sigma_{1}-\sigma_{2}\right)^{2}+\left(\sigma_{2}-\sigma_{3}\right)^{2}+\left(\sigma_{1}-\sigma_{3}\right)^{2}\right)
$$

where $\sigma_{1}, \sigma_{2}, \sigma_{3}$ denote the principal stress components of $\sigma$. Hence, the yield surface for the von Mises criteria is a cylinder of radius $\sqrt{\frac{2}{3}} \sigma_{y}$ with the axe $\sigma_{1}=\sigma_{2}=\sigma_{3}$

\subsection{Kinematics}

Following [16], the total deformation is the composition of a plastic and an elastic deformation (see Fig 1).

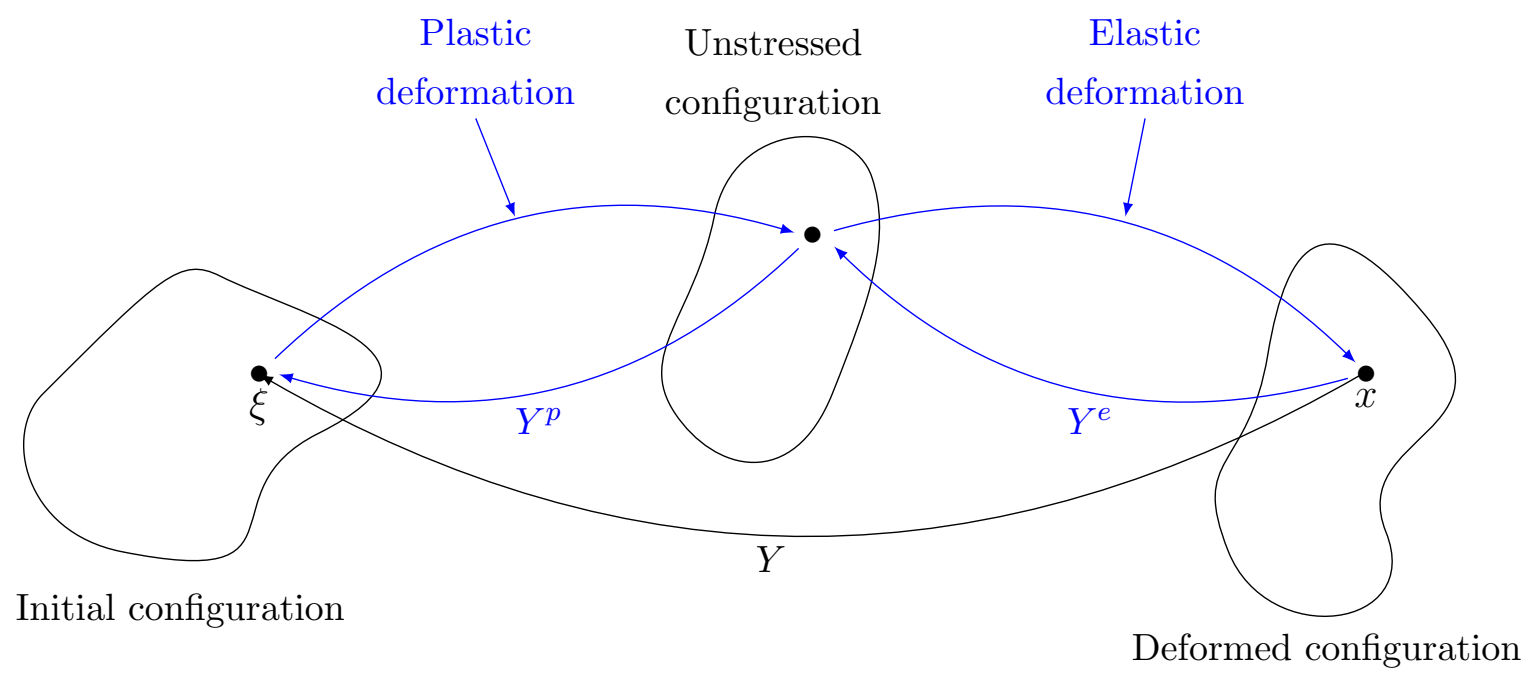

Fig. 1. Elastic and plastic deformations

The backward characteristics for elastic and plastic deformations are denoted 
by $Y^{e}$ and $Y^{p}$, respectively. Taking the gradient of the relation $Y(x, t)=$ $Y^{p}\left(Y^{e}(x, t), t\right)$ we get $\left[\nabla_{x} Y\right]=\left[\nabla_{x} Y^{p}\right]\left[\nabla_{x} Y^{e}\right]$ and the total deformation gradient $F$ is given by

$$
F:=\left[\nabla_{x} Y\right]^{-1}=\left[\nabla_{x} Y^{e}\right]^{-1}\left[\nabla_{x} Y^{p}\right]^{-1}:=F^{e} F^{p}
$$

which is known in the literature as the multiplicative decomposition of the total deformation gradient. We observe that after unloading the elastic force, $F^{e}=$ Id and $F=F^{p}$ so the unstressed reference state has changed. In the following we use the notation $\nabla$ for $\nabla_{x}$.

Next, we derive the Eulerian form of the evolution equation for the plastic deformation tensor $F^{p}$ :

$$
\partial_{t}\left(F^{p}\right)+u \cdot \nabla F^{p}=L^{p} F^{p}
$$

where $L^{p}$ is the constitutive law defining the plastic deformation rate. The equation on deformations in $(1)$ reads $\partial_{t}\left(F^{-1}\right)+\nabla\left(u \cdot F^{-1}\right)=0$ and hence

$$
\partial_{t}\left(F^{-1}\right)+u \cdot \nabla F^{-1}=-F^{-1}[\nabla u]
$$

Following (11) and (12) we get that $\left[\nabla Y^{e}\right]=\left[F^{e}\right]^{-1}=F^{p} F^{-1}$ verifies

$$
\partial_{t}\left(\nabla Y^{e}\right)+\nabla\left(u \cdot \nabla Y^{e}\right)=L^{p}\left[\nabla Y^{e}\right]
$$

We model plasticity by the constitutive law [18]

$$
L^{p}=\frac{1}{\chi \tau}\left[\nabla Y^{e}\right] \operatorname{dev}(\sigma)\left[\nabla Y^{e}\right]^{-1}
$$

where $\chi$ is the shear modulus and $\tau$ is time of relaxation of the plastic process which will be defined in the following in (25). Finally, the equations of conservation with plasticity modelling are 


$$
\left\{\begin{aligned}
\partial_{t} \rho+\nabla \cdot(\rho u) & =0 \\
\partial_{t}(\rho u)+\nabla \cdot(\rho u \otimes u-\sigma) & =0 \\
\partial_{t}\left(\nabla Y^{e}\right)+\nabla\left(u \cdot \nabla Y^{e}\right) & =\frac{1}{\chi \tau}\left[\nabla Y^{e}\right] \operatorname{dev}(\sigma) \\
\partial_{t}(\rho e)+\nabla \cdot\left(\rho e u-\sigma^{\top} u\right) & =0
\end{aligned}\right.
$$

The plasticity appears as a source term in the equation of elastic deformations and can be seen as a penalization of the deviatoric part of $\sigma$.

\subsection{Volume change in the plastic process}

We get with the relation $\partial_{t}(\operatorname{det}(A))=\operatorname{det}(A) \operatorname{Tr}\left(A^{-1} \partial_{t} A\right)$, (11), (14) and $\operatorname{Tr}(\operatorname{dev}(\sigma))=0$ that

$$
\begin{array}{r}
\frac{\mathrm{d}}{\mathrm{d} t}\left(\operatorname{det}\left(F^{p}\right)\right):=\partial_{t}\left(\operatorname{det}\left(F^{p}\right)\right)+u \cdot \nabla\left(\operatorname{det}\left(F^{p}\right)\right)= \\
\operatorname{det}\left(F^{p}\right) \operatorname{Tr}\left(\left[F^{p}\right]^{-1} \frac{\left[F^{e}\right]^{-1} \operatorname{dev}(\sigma)}{\chi \tau} F^{e} F^{p}\right)=0
\end{array}
$$

Hence, the plasticity relaxation occurs with no variation of volume.

\subsection{Clausius-Duhem inequality}

We assume that internal energy depends only on elastic deformations and entropy. Using $\varepsilon=e-\frac{1}{2}|u|^{2}$ and the equation on momentum conservation, the energy equation in (15) in the non adiabatic case can be replaced by

$$
\rho\left(\partial_{t} \varepsilon+u \cdot \nabla \varepsilon\right)=\sigma:[\nabla u]-\operatorname{div}(q)
$$

where $q$ is the heat flux. Internal energy is given by $\varepsilon=\varepsilon\left(F^{e}, s\right)$ so that

$$
\rho \frac{\partial \varepsilon}{\partial s}\left(\partial_{t} s+u \cdot \nabla s\right)+\underbrace{\rho \frac{\partial \varepsilon}{\partial F^{e}}:\left(\partial_{t}\left(F^{e}\right)+u \cdot \nabla F^{e}\right)}_{\mathcal{D}}=\sigma:[\nabla u]
$$


From (13), recalling that $F^{e}=\left[\nabla Y^{e}\right]^{-1}$ and that $\partial_{t}\left(A^{-1}\right)=-A^{-1} \partial_{t}(A) A^{-1}$, we have

$$
\partial_{t}\left(F^{e}\right)+u \cdot \nabla F^{e}=[\nabla u] F^{e}-\frac{1}{\chi \tau} \operatorname{dev}(\sigma) F^{e}
$$

and hence

$$
\mathcal{D}=\rho \frac{\partial \varepsilon}{\partial F^{e}}:\left([\nabla u] F^{e}-\frac{1}{\chi \tau} \operatorname{dev}(\sigma) F^{e}\right)
$$

Combining (16) with the following entropy inequality

$$
\rho T\left(\partial_{t} s+u \cdot \nabla s\right) \geq-\operatorname{div}(q)+\frac{q \cdot \nabla T}{T}
$$

we obtain the adiabatic Clausius-Duhem inequality $(q=0)$

$$
\begin{aligned}
& \rho\left(\mathrm{T}-\frac{\partial \varepsilon}{\partial s}\right)\left(\partial_{t} s+u \cdot \nabla s\right)+\left(\sigma-\rho \frac{\partial \varepsilon}{\partial F^{e}}\left[F^{e}\right]^{T}\right):[\nabla u]+ \\
& \frac{1}{\chi \tau} \rho \frac{\partial \varepsilon}{\partial F^{e}}\left[F^{e}\right]^{T}: \operatorname{dev}(\sigma) \geq 0
\end{aligned}
$$

that holds for any function $s(x, t), u(x, t)$ and $F^{e}(x, t)$. In order to satisfy this inequality, the stress tensor has to be $\sigma=\rho \frac{\partial \varepsilon}{\partial F^{e}}\left[F^{e}\right]^{T}$ and the temperature $T=\frac{\partial \varepsilon}{\partial s}$. Therefore, since $\sigma: \operatorname{dev}(\sigma)=|\operatorname{dev}(\sigma)|^{2} \geq 0$, the Clausius-Duhem inequality is indeed verified.

We remark that the expression for the Cauchy stress tensor only involves $\nabla Y^{e}$, that in turn satisfies the third equation in (15). If the unstressed configuration and the plastic deformations are sought, we need to integrate the nine equations (11) or equivalently the nine equations on the total deformation $\partial_{t}(\nabla Y)+\nabla(u \cdot \nabla Y)=0$.

In summary, when plasticity occurs thermodynamic consistency is ensured since entropy is increasing. 


\subsection{Stability of the deviator for the neohookean law}

Let us show that for the neohookean law, the norm of the deviator decreases when plasticity occurs. We consider a spatially homogeneous case for $u$ and $\nabla \tilde{Y}^{e}$, so that the deformation equation reduces to

$$
\partial_{t}\left(\nabla \tilde{Y}^{e}\right)=\frac{1}{\chi \tau}\left[\nabla \tilde{Y}^{e}\right] \operatorname{dev}(\tilde{\sigma})
$$

For the neohookean model

$$
\operatorname{dev}(\tilde{\sigma})=2 \chi \tilde{J}^{-5 / 3}\left(\tilde{B}-\frac{\operatorname{Tr}(\tilde{B})}{3} I\right)
$$

where $\tilde{B}=\left[\nabla \tilde{Y}^{e}\right]^{-1}\left[\nabla \tilde{Y}^{e}\right]^{-T}$ and $\tilde{J}=\operatorname{det}\left(\left[\nabla \tilde{Y}^{e}\right]\right)$. We have with (19)

$$
\partial_{t}\left(\left[\nabla \tilde{Y}^{e}\right]^{-1}\right)=-\frac{1}{\chi \tau} \operatorname{dev}(\tilde{\sigma})\left[\nabla \tilde{Y}^{e}\right]^{-1} \quad \partial_{t}\left(\left[\nabla \tilde{Y}^{e}\right]^{-T}\right)=-\frac{1}{\chi \tau}\left[\nabla \tilde{Y}^{e}\right]^{-T} \operatorname{dev}(\tilde{\sigma})
$$

Therefore because $\operatorname{dev}(\tilde{\sigma})$ is a polynomial in $\tilde{B}$

$$
\partial_{t} \tilde{B}=-\frac{2}{\chi \tau} \operatorname{dev}(\tilde{\sigma}) \tilde{B}=-\frac{2}{\chi \tau} \tilde{B} \operatorname{dev}(\tilde{\sigma})
$$

We get also with $\partial_{t}(\operatorname{det}(A))=\operatorname{det}(A) \operatorname{Tr}\left(A^{-1} \partial_{t} A\right),(19)$ and $\operatorname{Tr}(\operatorname{dev}(\tilde{\sigma}))=0$ that

$$
\partial_{t} \tilde{J}=\operatorname{det}\left(\nabla \tilde{Y}^{e}\right) \operatorname{Tr}\left(\left[\nabla \tilde{Y}^{e}\right]^{-1} \partial_{t}\left(\nabla \tilde{Y}^{e}\right)\right)=\frac{1}{\chi \tau} \operatorname{det}\left(\nabla \tilde{Y}^{e}\right) \operatorname{Tr}(\operatorname{dev}(\tilde{\sigma}))=0
$$

Hence $\tilde{J}$ is constant in time. Using (20), (22) and (23) 


$$
\begin{aligned}
\partial_{t}\left(|\operatorname{dev}(\tilde{\sigma})|^{2}\right) & =-\frac{8 \tilde{J}^{-5 / 3}}{\tau}(\operatorname{dev}(\tilde{\sigma}):(\tilde{B} \operatorname{dev}(\tilde{\sigma}))-\underbrace{\frac{\operatorname{Tr}(\tilde{B} \operatorname{dev}(\tilde{\sigma}))}{3} \operatorname{dev}(\tilde{\sigma}): I}_{=0 \text { because } \operatorname{Tr}(\operatorname{dev}(\tilde{\sigma}))=0}) \\
& =-\frac{8 \tilde{J}^{-5 / 3}}{\tau}\left(2 \chi \tilde{J}^{-5 / 3}\right)^{2}\left(\tilde{B}-\frac{\operatorname{Tr}(\tilde{B})}{3} I\right):\left(\tilde{B}^{2}-\frac{\operatorname{Tr}(\tilde{B})}{3} \tilde{B}\right) \\
& =-\frac{32 \tilde{J}^{-5} \chi^{2}}{9 \tau} \underbrace{\left(9 \operatorname{Tr}(\tilde{B})^{3}-6 \operatorname{Tr}(\tilde{B}) \operatorname{Tr}\left(\tilde{B}^{2}\right)+\operatorname{Tr}(\tilde{B})^{3}\right)}_{\mathcal{P}}
\end{aligned}
$$

The matrix $\tilde{B}$ is symmetric and positive definite since $(\tilde{B} x) \cdot x=\left|\left[\nabla \tilde{Y}^{e}\right]^{-T} x\right|^{2} \geq$ 0 so $\tilde{B}$ is diagonalizable in an orthonormal basis and the eigenvalues denoted by $\lambda_{i}$ are positives. We have that

$$
\begin{aligned}
\mathcal{P} & =5\left(\left(\lambda_{1}\right)^{3}+\left(\lambda_{2}\right)^{3}+\left(\lambda_{3}\right)^{3}\right)+12 \lambda_{1} \lambda_{2} \lambda_{3}-\left(\lambda_{1}+\lambda_{2}+\lambda_{3}\right)^{3} \\
& =\lambda_{1}\left(-2 \lambda_{1}+\lambda_{2}+\lambda_{3}\right)^{2}+\lambda_{2}\left(\lambda_{1}-2 \lambda_{2}+\lambda_{3}\right)^{2}+\lambda_{3}\left(\lambda_{1}+\lambda_{2}-2 \lambda_{3}\right)^{2} \geq 0
\end{aligned}
$$

Hence

$$
\partial_{t}\left(|\operatorname{dev}(\tilde{\sigma})|^{2}\right) \leq 0
$$

Here we use the relaxation time given in [7]:

$$
\frac{1}{\tau}=\left\{\begin{array}{lll}
\frac{1}{\tau_{0}} \frac{|\operatorname{dev}(\sigma)|^{2}-\frac{2}{3}\left(\sigma_{y}\right)^{2}}{\chi^{2}} & \text { if } & f_{V M}(\sigma)>0 \\
0 & \text { if } & f_{V M}(\sigma) \leq 0
\end{array}\right.
$$

where $\tau_{0}$ is a characteristic relaxation time. Therefore, during the plastic process the quantity $|\operatorname{dev}(\sigma)|$ is decreasing until reaching the yield surface of plasticity at constant pressure $(p=p(\tilde{J}, s))$. 


\section{Numerical Scheme}

A splitting strategy is used at each time step. First, we compute the solution of the equations (15) without the plastic source term. For sake of completeness we report here the main ideas of the hyperbolic part of the scheme. Details can be found in $[13,11,5]$.

\subsection{Hyperbolic step}

We use a finite-volume method on a Cartesian mesh and the fluxes are computed by approximate one-dimensional Riemann solvers in the direction orthogonal to the cell sides of the Cartesian mesh. The numerical flux function $\mathcal{F}\left(\Psi_{l} ; \Psi_{r}\right)$ at the cell interface $x=0$ is determined based on the solution of the HLLC [26] approximate Riemann problem. Even though the exact wave pattern involves seven distinct waves, see (6), the approximate solver approaches the solution using three waves: the contact discontinuity $u_{1}^{\star}$, the fastest leftward and rightward waves $s_{l}$ and $s_{r}$. Hence, there exist only two intermediate states $\Psi^{-}$and $\Psi^{+}$(see Fig. 2).

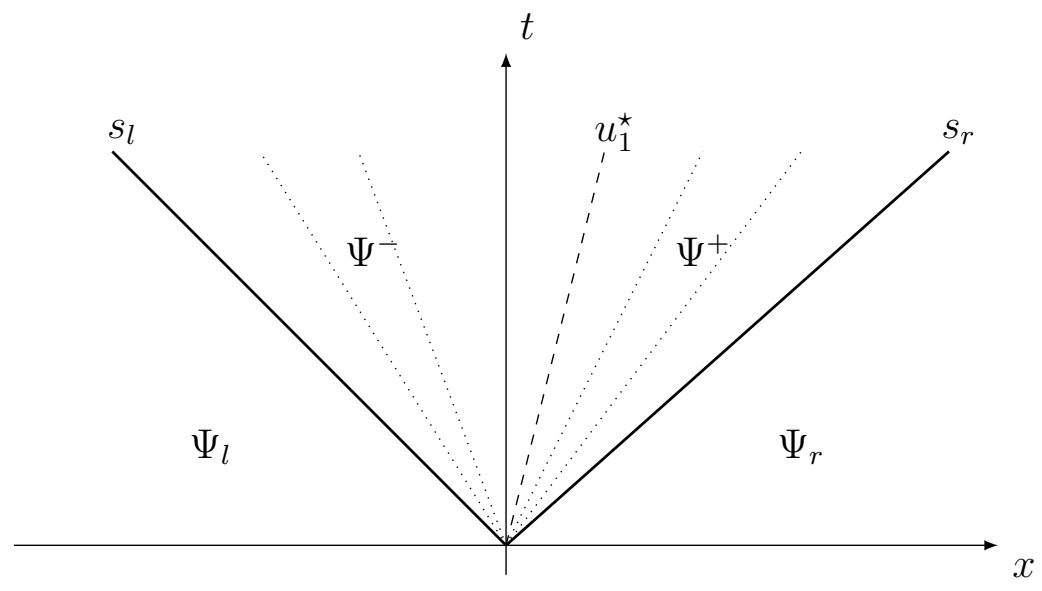

Fig. 2. HLLC solver wave pattern.

The HLLC scheme is based on the assumption that every wave is a shock and therefore Rankine-Hugoniot relations give 


$$
\left\{\begin{aligned}
F\left(\Psi_{r}\right)-\mathcal{F}^{+} & =s_{r}\left(\Psi_{r}-\Psi^{+}\right) \\
\mathcal{F}^{+}-\mathcal{F}^{-} & =u_{1}\left(\Psi^{+}-\Psi^{-}\right) \\
\mathcal{F}^{-}-F\left(\Psi_{l}\right) & =s_{l}\left(\Psi^{-}-\Psi_{l}\right)
\end{aligned}\right.
$$

These relations allow to compute the intermediate states $\Psi^{-}$and $\Psi^{+}$. The robustness of the scheme is strongly influenced by the estimation of $s_{l}$ and $s_{r}$. We use the estimate presented in [4] which is a simple way to obtain robust speed estimates :

$$
s_{l}=\min \left(\left(u_{1}-\lambda\right)_{l},\left(u_{1}-\lambda\right)_{r}\right) \quad s_{r}=\max \left(\left(u_{1}+\lambda\right)_{l},\left(u_{1}+\lambda\right)_{r}\right) .
$$

where $\lambda=\sqrt{\frac{\max \alpha_{i}}{\rho}}$. The numerical flux at the cell interface $x=0$ is then given by

$$
\mathcal{F}\left(\Psi_{l} ; \Psi_{r}\right)=\left\{\begin{array}{rlc}
F\left(\Psi_{l}\right) & \text { if } & 0 \leq s_{l} \\
\mathcal{F}^{-} & \text {if } & s_{l} \leq 0 \leq u_{1}^{\star} \\
\mathcal{F}^{+} & \text {if } & u_{1}^{\star} \leq 0 \leq s_{r} \\
F\left(\Psi_{r}\right) & \text { if } & s_{r} \leq 0
\end{array}\right.
$$

The multimaterial solver is detailed in one dimension for sake of clarity and the same method in all directions. We consider a case where the interface separating materials with different constitutive laws is located between the cell centers $k-1$ and $k$. The main idea of the multimaterial solver is that, instead of (27) we take (see Fig. 3)

$$
\mathcal{F}_{k-1 / 2}^{l}=\mathcal{F}^{-} \quad \mathcal{F}_{k-1 / 2}^{r}=\mathcal{F}^{+}
$$




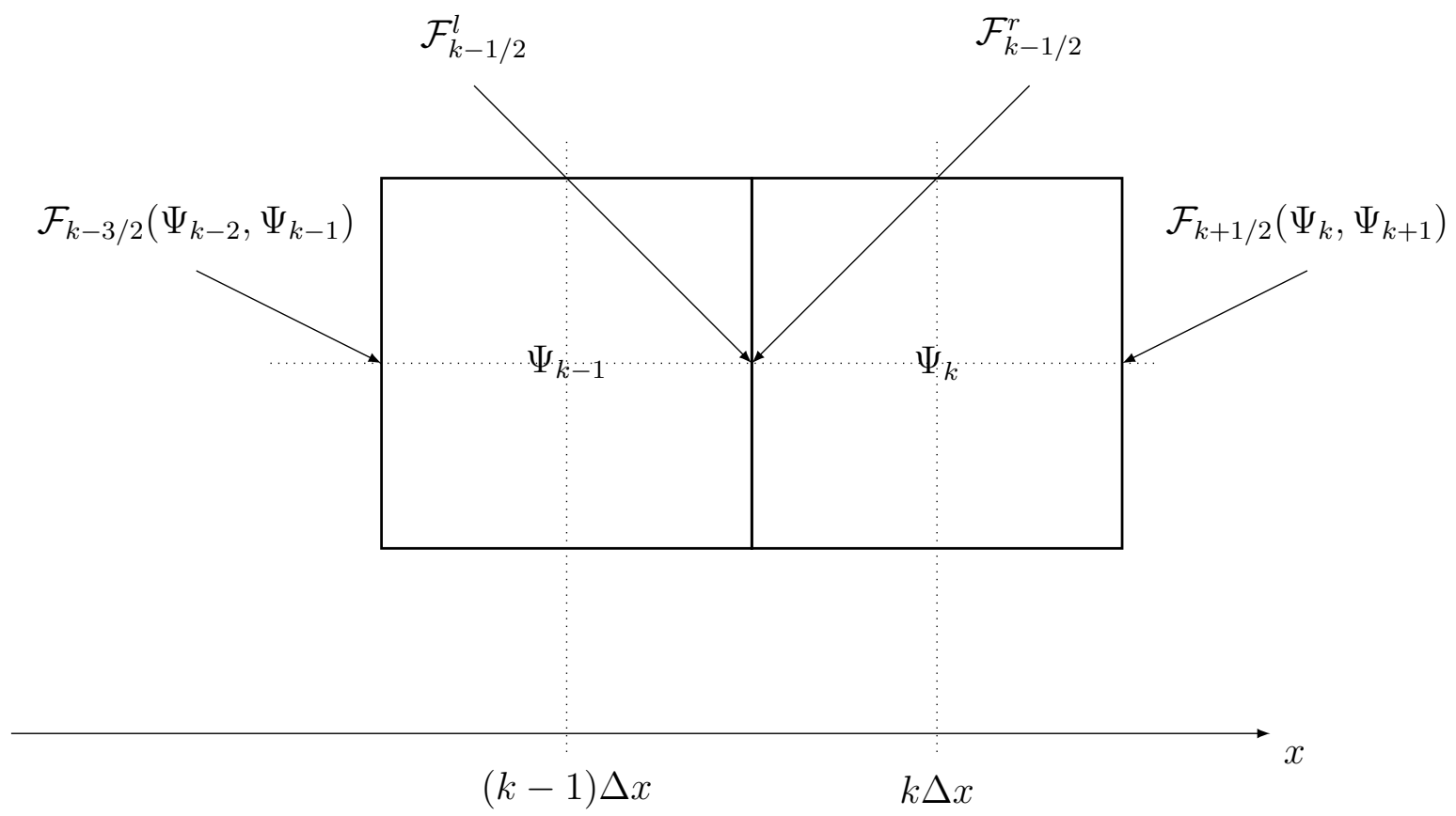

Fig. 3. Fluxes at the material discontinuity.

The scheme is extended to second-order accuracy using a piecewise-linear slope reconstruction in space (MUSCL), with minmod limiter. With this scheme the interface remains sharp.

Coherently with a fully Eulerian approach, a level set function is used to follow the interface separating different materials. The level set function is transported with the velocity field by the equation :

$$
\varphi_{t}+u \cdot \nabla \varphi=0
$$

This equation is approximated with a WENO 5 scheme [14]. The conservation equations and the interface advection are explicitly integrated in time by a Runge-Kutta 2 scheme. The interface position is advected using the material velocity field. For numerical stability, the integration step is limited by the fastest characteristics over the grid points. 


\subsection{Plastic step}

Secondly, we solve the plastic ODE

$$
\partial_{t}\left(\nabla Y^{e}\right)=\frac{1}{\chi \tau}\left[\nabla Y^{e}\right] \operatorname{dev}(\sigma)
$$

If we use a explicit Euler scheme, the time step will be limited by a CFL condition of the type $\Delta t \leq C \chi \tau$ which is very restrictive. Instead we use the following semi-implicit scheme. The term $\operatorname{dev}(\sigma)$ is taken explicitly at time $t_{n}$ and the term $\left[\nabla Y^{e}\right]$ is chosen implicitly so the solution at time $t_{n+1}$ is given analytically by

$$
\left[\nabla Y^{e}\right]^{n+1}=\left[\nabla Y^{e}\right]^{n} \exp \left(\frac{\Delta t}{\chi \tau} \operatorname{dev}\left(\sigma^{n}\right)\right)
$$

\section{$5 \quad$ Numerical results}

We present in this section simulations of elasto-plastic problems in one, two and three dimensions. The code is fully parallelized with the MPI paradigm. It is used with one grid cell in the directions $y$ and $z$ for one-dimensional simulations, and similarly for two-dimensional cases.

\section{$5.11 D$ validation with analytical solution}

In $\mathrm{TC} 1$, an aluminium plate of $2 \mathrm{~mm}$ impacts a titanium plate of $9.8 \mathrm{~mm}$. This case was presented in [21]. The initial velocity of the aluminium plate is $700 \mathrm{~m} . \mathrm{s}^{-1}$. The computational domain is $[0,17] \mathrm{mm}$ where the intervals $[0,1] \mathrm{mm}$ and $[12.8,17] \mathrm{mm}$ are filled with air. The initial configuration is presented in Fig 4 and the physical parameters in Tab 1. The computation is performed on 2000 points with a CFL of 0.8 . 


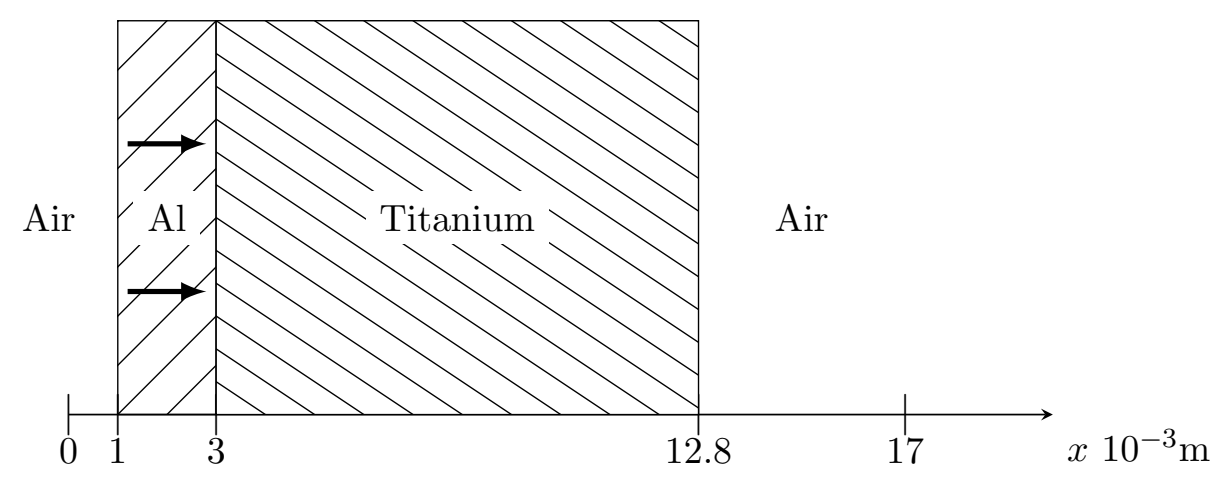

Fig. 4. Sketch of the initial configuration for TC1

\begin{tabular}{|l|c|c|c|c|c|c|}
\hline Media & $\rho\left(k g . \mathrm{m}^{-3}\right)$ & $\gamma$ & $p_{\infty}(\mathrm{GPa})$ & $\chi(\mathrm{GPa})$ & $\sigma_{y}(\mathrm{GPa})$ & $\tau_{0}(\mathrm{~s})$ \\
\hline Air & 1 & 1.4 & 0 & 0 & - & - \\
\hline Aluminium (Al) & 2712 & 3.5 & 32 & 26 & 0.06 & $10^{-9}$ \\
\hline Titanium & 4527 & 2.6 & 44 & 42 & 1.03 & $10^{-8}$ \\
\hline
\end{tabular}

\section{Table 1}

Material physical parameters of TC1

After the impact, two waves are created: an elastic shock wave named elastic precursor and a plastic region propagating with a smaller velocity. The wave pattern in space-time of this elasto-plastic phenomenon is sketched on Fig 5, where the plastic region is designated by a single plastic wave. The plastic wave represents the region where the stress of the material is relaxing towards the plasticity surface.

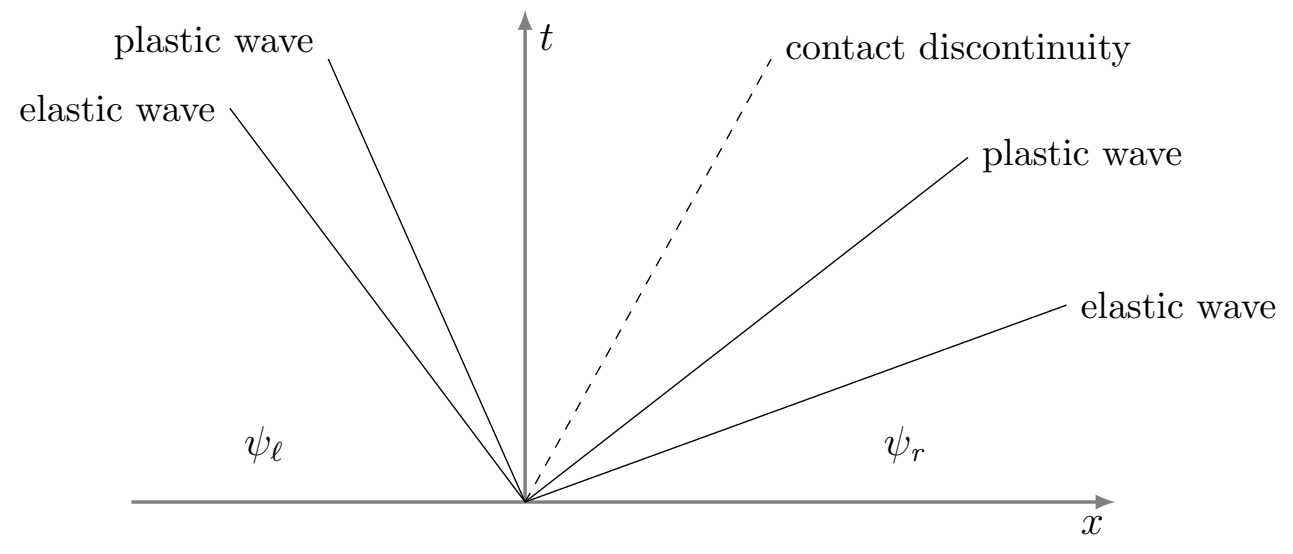

Fig. 5. Solution pattern of elasto-plastic problem.

Fig 6 presents the numerical results for the density, pressure, velocity and 
normal stress at $t=0.2 \mu \mathrm{s}$. We can see the plastic wave at $x=4 \mathrm{~mm}$ and the elastic wave at $x=4.5 \mathrm{~mm}$ that propagate in titanium. These waves are less distinguishable in the aluminium plate because the plastic yield limit $\left(\sigma_{y}=0.06 \mathrm{GPa}\right)$ is reached almost immediately. However, we can see on the pressure plot the elastic wave at $x=1.2 \mathrm{~mm}$ and the plastic wave at $x=1.6 \mathrm{~mm}$. The results found in this work are similar to those observed in [21] where a different constitutive law is used.
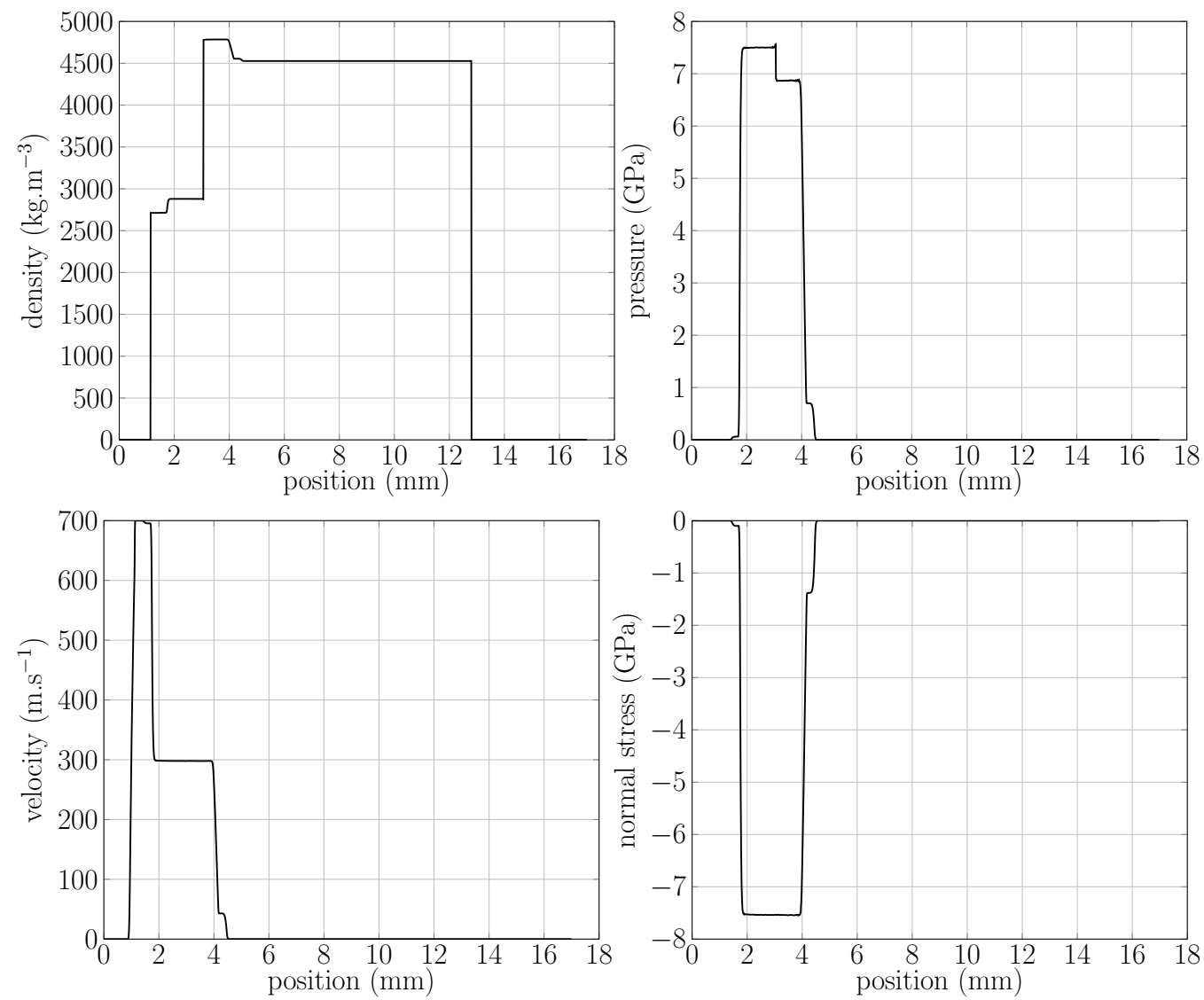

Fig. 6. TC1: Solution at $t=0.2 \mu \mathrm{s}$ for density, pressure, velocity and normal stress

In order to validate the numerical scheme, the analysis done in [21] is adapted to the neohookean model. The problem is $1 \mathrm{D}$ and the initial condition on velocity is $u=\left(u_{1}, 0,0\right)$, so $[\nabla Y]$ is diagonal for all times and hence 


$$
[\nabla Y]=\left(\begin{array}{ccc}
Y_{, 1}^{1} & 0 & 0 \\
0 & Y_{, 2}^{2} & 0 \\
0 & 0 & Y_{, 3}^{3}
\end{array}\right)
$$

Even if the problem is $1 \mathrm{D}$ in the $x_{1}$ direction, the three components of the diagonal matrix $[\nabla Y]$ are modified in the plastic regime because $\operatorname{dev}(\sigma)$ is a non linear function of $[\nabla Y]$. The density $\rho$ is defined by $\rho=\rho_{0} Y_{, 1}^{1} Y_{, 2}^{2} Y_{, 3}^{3}$. For the neohookean model, the internal energy is given by

$\varepsilon=\frac{p+\gamma p_{\infty}}{(\gamma-1) \rho_{0} Y_{, 1}^{1} Y_{, 2}^{2} Y_{, 3}^{3}}+\frac{\chi}{\rho_{0}}\left(\left(Y_{, 1}^{1} Y_{, 2}^{2} Y_{, 3}^{3}\right)^{-\frac{4}{3}}\left(\left(Y_{, 1}^{1}\right)^{2}\left(Y_{, 2}^{2}\right)^{2}+\left(Y_{, 1}^{1}\right)^{2}\left(Y_{, 3}^{3}\right)^{2}+\left(Y_{, 2}^{2}\right)^{2}\left(Y_{, 3}^{3}\right)^{2}\right)-3\right)$

whereas the deviatoric of the Cauchy stress tensor is diagonal and its components are given by

$$
\begin{aligned}
{[\operatorname{dev}(\sigma)]_{11} } & =\frac{2 \chi}{3\left(Y_{, 1}^{1} Y_{, 2}^{2} Y_{, 3}^{3}\right)^{\frac{1}{3}}}\left(2\left(Y_{, 2}^{2}\right)^{2}\left(Y_{, 3}^{3}\right)^{2}-\left(Y_{, 1}^{1}\right)^{2}\left(\left(Y_{, 2}^{2}\right)^{2}+\left(Y_{, 3}^{3}\right)^{2}\right)\right) \\
{[\operatorname{dev}(\sigma)]_{22} } & =\frac{2 \chi}{3\left(Y_{, 1}^{1} Y_{, 2}^{2} Y_{, 3}^{3}\right)^{\frac{1}{3}}}\left(2\left(Y_{, 1}^{1}\right)^{2}\left(Y_{, 3}^{3}\right)^{2}-\left(Y_{, 2}^{2}\right)^{2}\left(\left(Y_{, 1}^{1}\right)^{2}+\left(Y_{, 3}^{3}\right)^{2}\right)\right) \\
{[\operatorname{dev}(\sigma)]_{33} } & =\frac{2 \chi}{3\left(Y_{, 1}^{1} Y_{, 2}^{2} Y_{, 3}^{3}\right)^{\frac{1}{3}}}\left(2\left(Y_{, 1}^{1}\right)^{2}\left(Y_{, 2}^{2}\right)^{2}-\left(Y_{, 3}^{3}\right)^{2}\left(\left(Y_{, 1}^{1}\right)^{2}+\left(Y_{, 2}^{2}\right)^{2}\right)\right)
\end{aligned}
$$

In this test case the material is not prestressed so $Y_{, 2}^{2}=Y_{, 3}^{3}=1$ at initial time.

Region between the elastic precursor and the plastic region. In this case the plastic source term vanishes so that $Y_{, 2}^{2}=Y_{, 3}^{3}=1$ and $\rho=\rho_{0} Y_{, 1}^{1}$. We suppose that $\sigma$ lays on the surface of plasticity in the elastic region so

$$
|\operatorname{dev}(\sigma)|^{2}=\frac{8 \chi^{2}}{3\left(Y_{, 1}^{1}\right)^{\frac{2}{3}}}\left(\left(Y_{, 1}^{1}\right)^{2}-1\right)^{2}=\frac{2}{3} \sigma_{y}^{2}
$$


The elastic wave is a shock wave so $Y_{, 1}^{1}>1$. Therefore, the equation becomes

$$
\left(Y_{, 1}^{1}\right)^{2}-\frac{\sigma_{y}}{2 \chi}\left(Y_{, 1}^{1}\right)^{\frac{1}{3}}-1=0
$$

The solution $Y_{, 1}^{1}$ of this equation is unique. Therefore, the density $\rho$ and the values of pressure $p$ and velocity $u$ can be computed with the Rankine-Hugoniot conditions. These values are reported for the aluminium and the titanium in Tab 2 along with the numerical results for different mesh sizes.

\begin{tabular}{|l|c|c|c|c|}
\hline Variables & \multirow{2}{*}{ analytical results } & \multicolumn{3}{|c|}{ numerical results } \\
\hline Mesh size & & 1000 & 2000 & 4000 \\
\hline$\rho_{\mathrm{Al}}\left(\mathrm{kg} \cdot \mathrm{m}^{-3}\right)$ & 2713.565 & 2713.476 & 2713.539 & 2713.540 \\
\hline$u_{\mathrm{Al}}\left({\left.\mathrm{m} . \mathrm{s}^{-1}\right)}^{-1}\right.$ & 695.283 & 695.544 & 695.352 & 695.349 \\
\hline$p_{\mathrm{Al}}(\mathrm{GPa})$ & 0.0648 & 0.0611 & 0.0637 & 0.0638 \\
\hline$\rho_{\mathrm{Ti}}\left(\mathrm{kg} \cdot \mathrm{m}^{-3}\right)$ & 4554.726 & 4554.332 & 4554.538 & 4554.643 \\
\hline$u_{\mathrm{Ti}}\left(\mathrm{m} . \mathrm{s}^{-1}\right)$ & 43.246 & 42.634 & 42.954 & 43.117 \\
\hline$p_{\mathrm{Ti}}(\mathrm{GPa})$ & 0.704 & 0.694 & 0.699 & 0.702 \\
\hline
\end{tabular}

Table 2

TC1: Analytic and numerical results for different mesh size in the elastic region for aluminium and titanium

Plastic plateau. In this case, the plastic source term does not vanish and hence $Y_{, 2}^{2}$ and $Y_{, 3}^{3}$ can be different from 1 . However, by symmetry $Y_{, 2}^{2}=Y_{, 3}^{3}$ and, therefore, $\rho=\rho_{0}\left(Y_{, 1}^{1}\right)\left(Y_{, 2}^{2}\right)^{2}$. After relaxation, $\sigma$ is on the surface of plasticity and hence

$$
|\operatorname{dev}(\sigma)|^{2}=\frac{8 \chi^{2}}{3\left(\left(Y_{, 1}^{1}\right)\left(Y_{, 2}^{2}\right)^{2}\right)^{\frac{2}{3}}}\left(Y_{, 2}^{2}\right)^{4}\left(\left(Y_{, 1}^{1}\right)^{2}-\left(Y_{, 2}^{2}\right)^{2}\right)^{2}=\frac{2}{3}\left(\sigma_{y}\right)^{2}
$$

The plastic relaxation region is now approximated by a shock wave. Hence, applying the Rankine-Hugoniot relations and using the assumption that the stress and the velocity are continuous across this wave, a system of 12 equations is obtained and is solved numerically. These values are reported for the 
aluminium and the titanium in Tab 3 along with the numerical results for different mesh sizes.

\begin{tabular}{|l|c|c|c|c|}
\hline Variables & analytical results & \multicolumn{3}{|c|}{ numerical results } \\
\hline Mesh size & & 1000 & 2000 & 4000 \\
\hline$\rho_{\mathrm{Al}}\left(\mathrm{kg} . \mathrm{m}^{-3}\right)$ & 2879.483 & 2879.057 & 2879.254 & 2879.383 \\
\hline$\rho_{\mathrm{Ti}}\left(\mathrm{kg} \cdot \mathrm{m}^{-3}\right)$ & 4784.050 & 4783.976 & 4778.498 & 4783.964 \\
\hline$u_{\text {Interface }}\left(\mathrm{m} . \mathrm{s}^{-1}\right)$ & 297.771 & 297.970 & 298.006 & 297.932 \\
\hline$\sigma_{\text {Interface }}^{11}(\mathrm{GPa})$ & -7.546 & -7.537 & -7.537 & -7.540 \\
\hline
\end{tabular}

Table 3

TC1: Analytic and numerical results for different mesh size in the plastic region for aluminium and titanium

We conclude that the numerical and analytical results are in good agreement $(<1 \%)$ in the elastic and plastic regions. The numerical results converge with the decrease of the mesh size in the elastic region.

\subsection{D Simulations}

We present two test cases of impacts of a projectile on a plate. In the first case the two media have different material properties whereas in the second one the materials are the same. In both cases, the projectile and the plate are represented by two different level set functions advected by a WENO5 scheme.

\subsubsection{Impact of iron on aluminium}

In TC2, an iron projectile impacts an aluminium plate immersed in air. The initial horizontal velocity of the iron projectile is $1000 \mathrm{~m} . \mathrm{s}^{-1}$. The initial configuration is presented in Fig 7 and the physical parameters in Tab 4. The computational domain is $[-0.3,0.7] \times[-0.4,0.4] \mathrm{m}^{2}$. The computation is performed on a $2000 \times 1600$ mesh with 144 processors. Homogeneous Neumann conditions are imposed on the left and right borders and cantilever conditions are imposed on the top and bottom. 


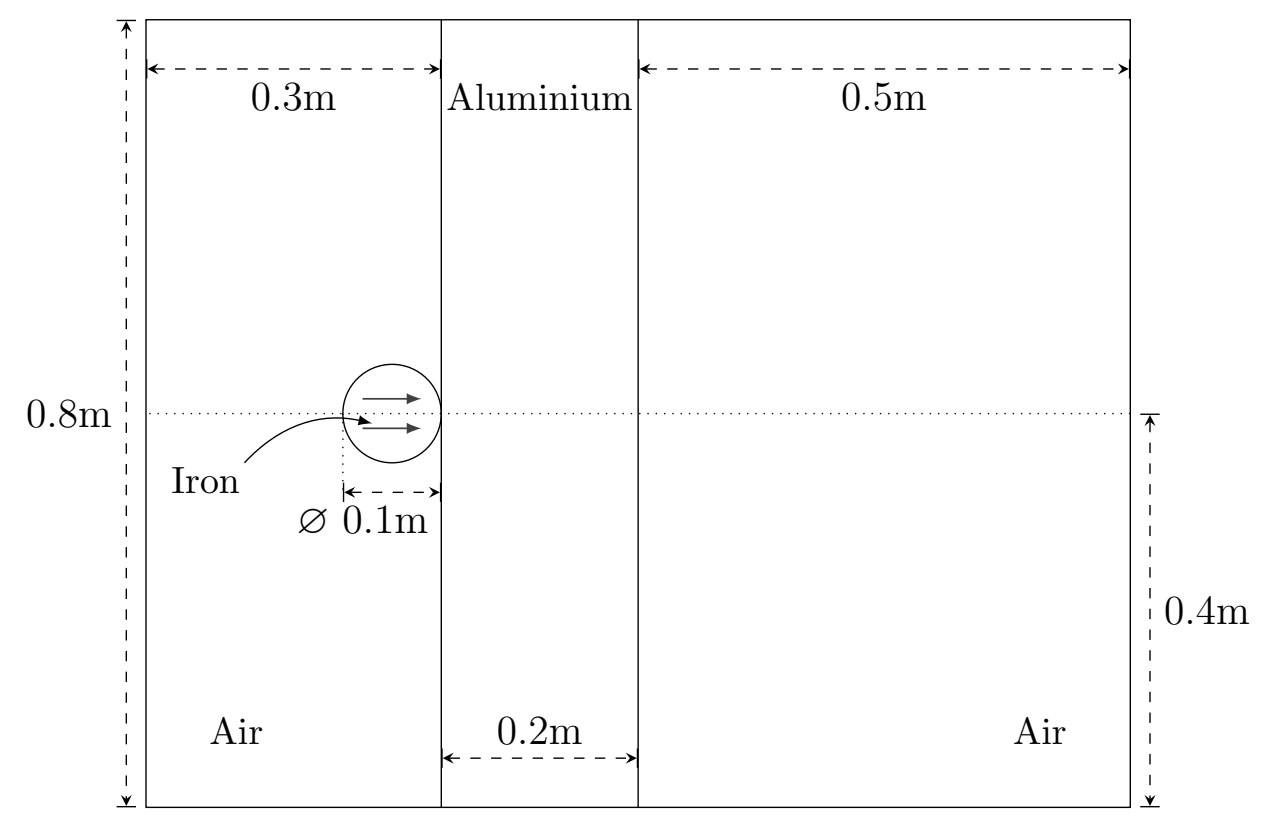

Fig. 7. Sketch of the initial configuration for TC2

\begin{tabular}{|l|c|c|c|c|c|c|}
\hline Media & $\rho\left(\mathrm{kg} \cdot \mathrm{m}^{-3}\right)$ & $\gamma$ & $p_{\infty}(\mathrm{GPa})$ & $\chi(\mathrm{GPa})$ & $\sigma_{y}(\mathrm{GPa})$ & $\tau_{0}(\mathrm{~s})$ \\
\hline Air & 1 & 1.4 & 0 & 0 & - & - \\
\hline Iron & 7860 & 3.9 & 43.6 & 82 & 0.2 & $2.10^{-6}$ \\
\hline Aluminium & 2712 & 3.5 & 32 & 26 & 0.06 & $3.10^{-7}$ \\
\hline
\end{tabular}

Table 4

Parameters of materials for TC2

The results are presented in Fig 8 with a Schlieren image (bottom) and the von Mises criteria $|\operatorname{dev}(\sigma)|^{2}-\frac{2}{3}\left(\sigma_{y}\right)^{2}$ (top) at different time steps. To plot the von Mises criteria, a log scale is used and the minimum value is fixed to $10^{9}$. Fig 8 a shows the shock waves that propagate into the aluminium target plate following the impact of the iron projectile on the plate. The Schlieren plot shows the longitudinal wave followed by the shear wave that induces the plastic deformation of the material. The waves reflect on the rear surface back into the plate (Fig 8b). As the waves travel back and forth in the aluminum plate, the successive reflections of the waves on the front and rear surface transmit waves in the air. Note that, in Fig 8f, the front wave generated in the air reflects on the top and bottom domain boundaries. The projectile penetrates into the plate. It deforms but does not flatten as much as in [25], 
where an aluminium projectile is used, because the yield plastic limit of iron is higher. The plate is strongly deformed and forms at the end a filament.

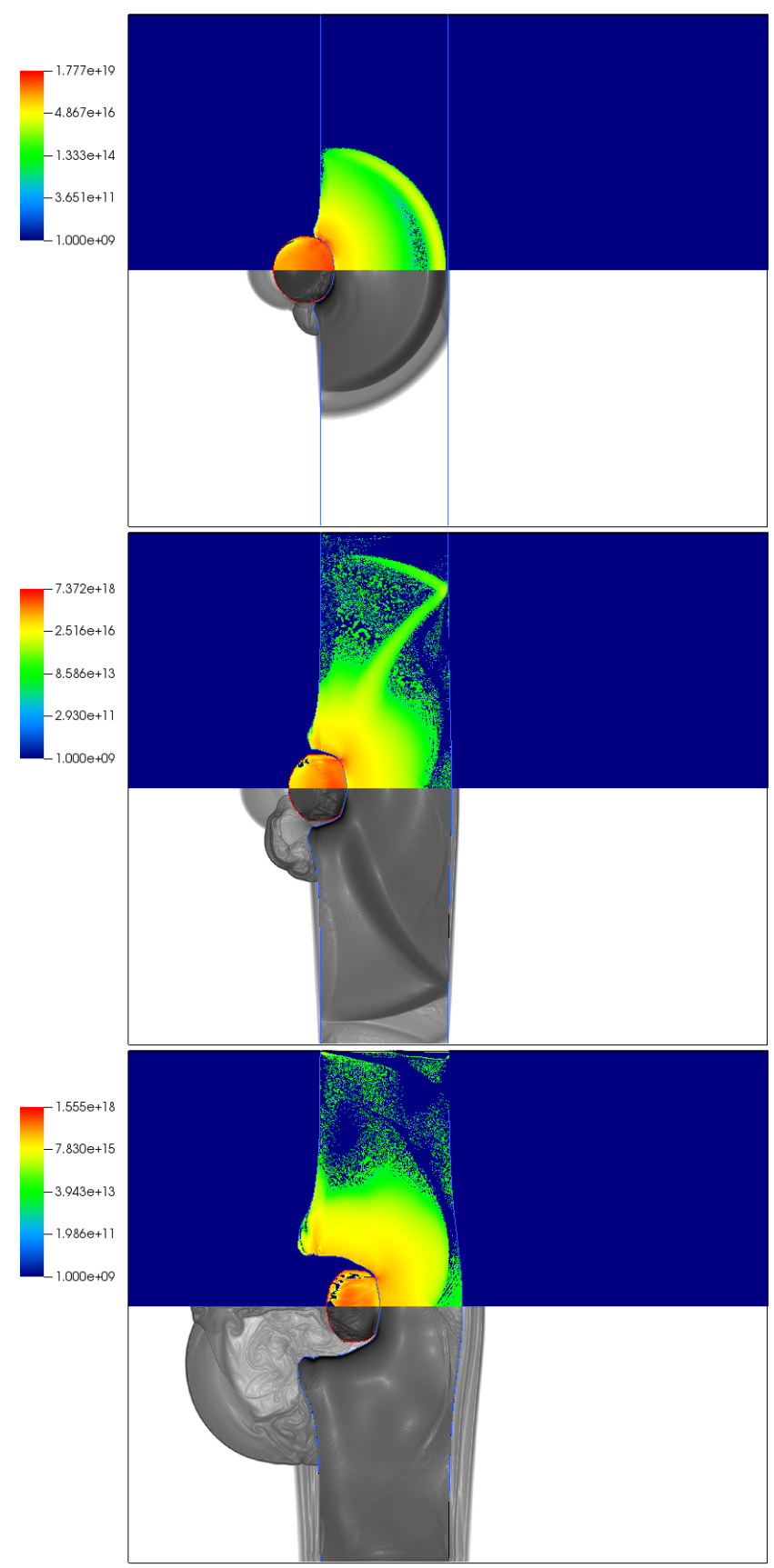




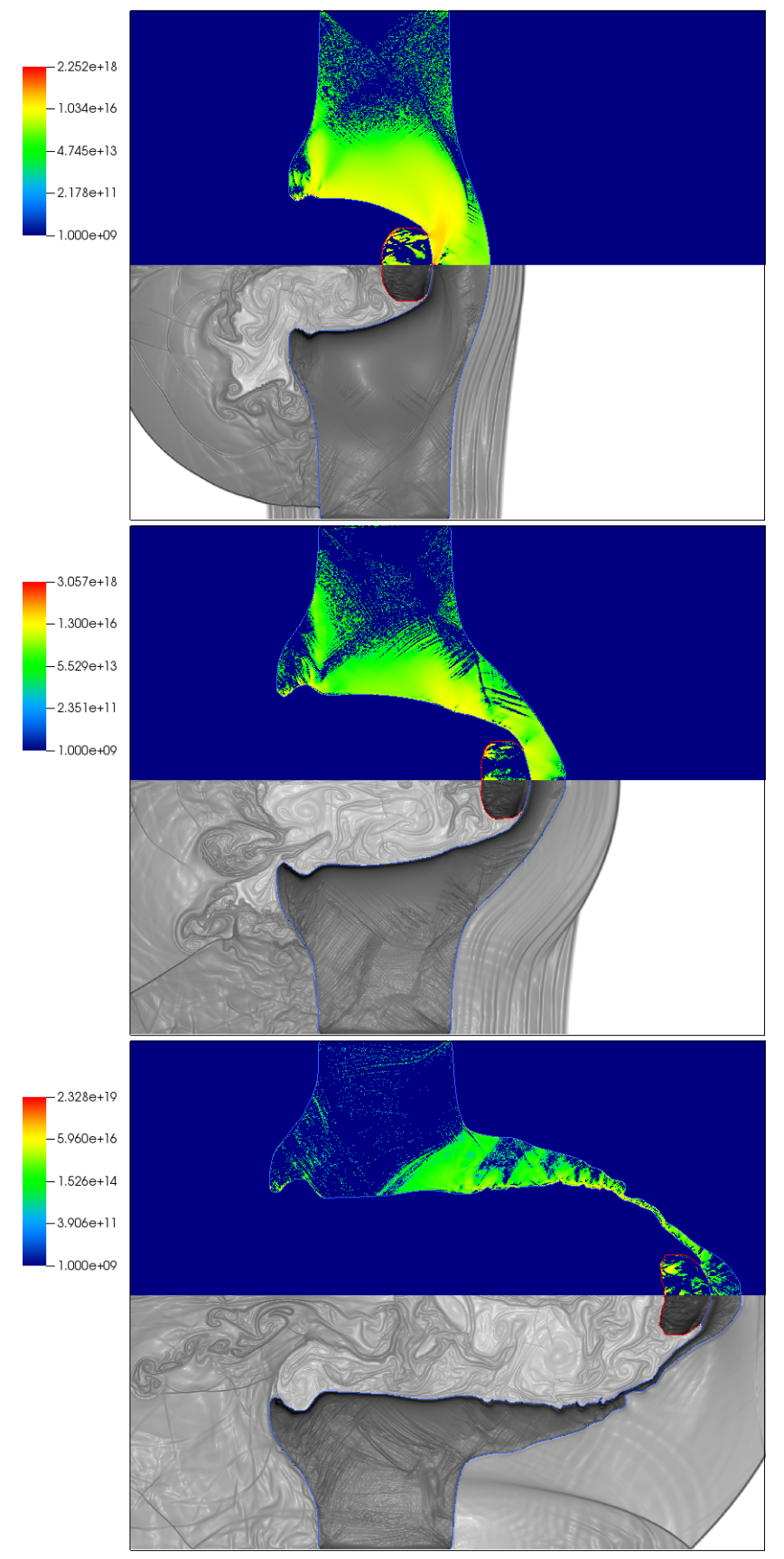

Fig. 8. TC2: Impact of iron on aluminium. Schlieren image and von Mises criteria at $t=0.03 \mathrm{~ms}, 0.06 \mathrm{~ms}, 0.13 \mathrm{~ms}, 0.26 \mathrm{~ms}, 0.53 \mathrm{~ms}$ and $t=1.04 \mathrm{~ms}$.

\subsubsection{Impact of copper on copper}

In TC3, a disc of copper impacts a plate of copper immersed in air. The initial horizontal velocity of the disc is $500 \mathrm{~m} . \mathrm{s}^{-1}$. The initial configuration is presented in Fig 9 and the physical parameters in Tab 5. The computational 
domain is $[-1,1] \times[-1,1] \mathrm{m}^{2}$. The computation is performed on a $2000 \times 2000$ mesh with 144 processors. Homogeneous Neumann conditions are imposed on the borders except for a symmetry condition on the right border.

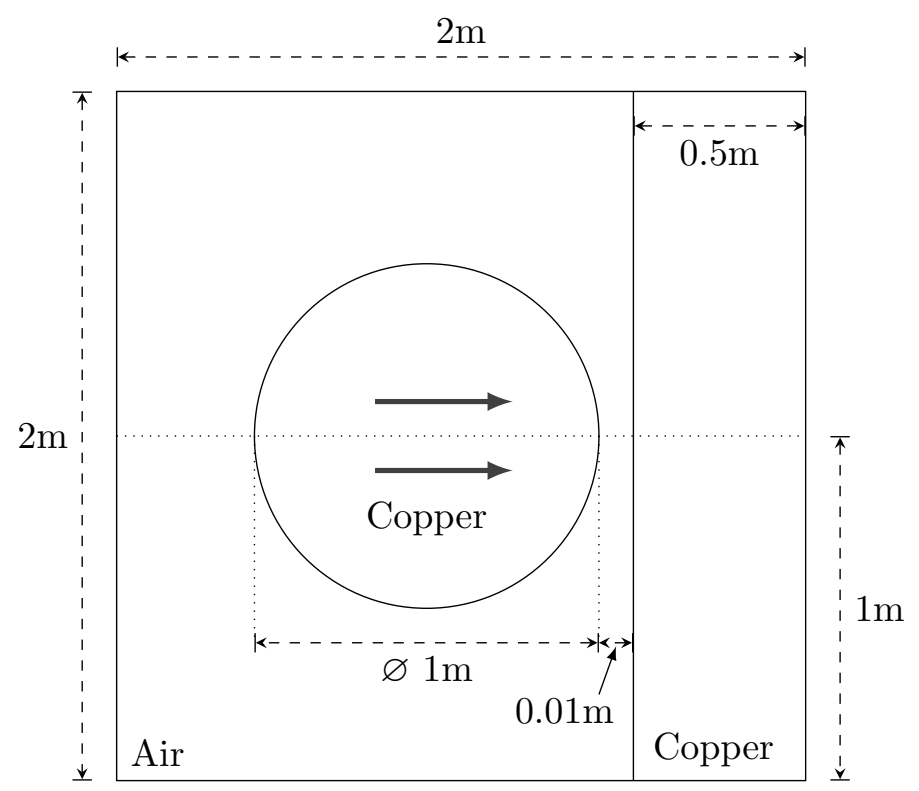

Fig. 9. Sketch of the initial configuration for TC3

Table 5

\begin{tabular}{|l|c|c|c|c|c|c|}
\hline Media & $\gamma$ & $\rho\left(\mathrm{kg} . \mathrm{m}^{-3}\right)$ & $p_{\infty}(\mathrm{GPa})$ & $\chi(\mathrm{GPa})$ & $\sigma_{y}(\mathrm{GPa})$ & $\tau_{0}(\mathrm{~s})$ \\
\hline Air & 1.4 & 1000 & 0 & 0 & - & - \\
\hline Copper & 4.22 & 8900 & 34.2 & 50 & 0.6 & $4.10^{-6}$ \\
\hline
\end{tabular}

Parameters of material for TC3

The results are presented in Fig 10 with a Schlieren image (bottom) and the von Mises criteria $|\operatorname{dev}(\sigma)|^{2}-\frac{2}{3}\left(\sigma_{y}\right)^{2}$ (top) at different time steps. The projectile impacts into the metal layer and symmetric compression and shear waves emerge in both the impactor and the plate, as the materials are similar. The waves reflect on the right domain boundary back into the plate. A strong jet of air is generated by the collision of the objects. The projectile deforms and tends to form a bulge. The von Mises criteria shows that the region where the plasticity effects are larger is at the triple point between the three materials and in the pleating of the deformed projectile. This is coherent with the deformation of the projectile and the formation of the bulge as the rest of the body undergoes less plastic strain. Reciprocally, the plate follows the 
deformation of the impactor. A striated pattern of the waves is observed in both the projectile and the plate. The von Mises criteria highlights the shear waves.
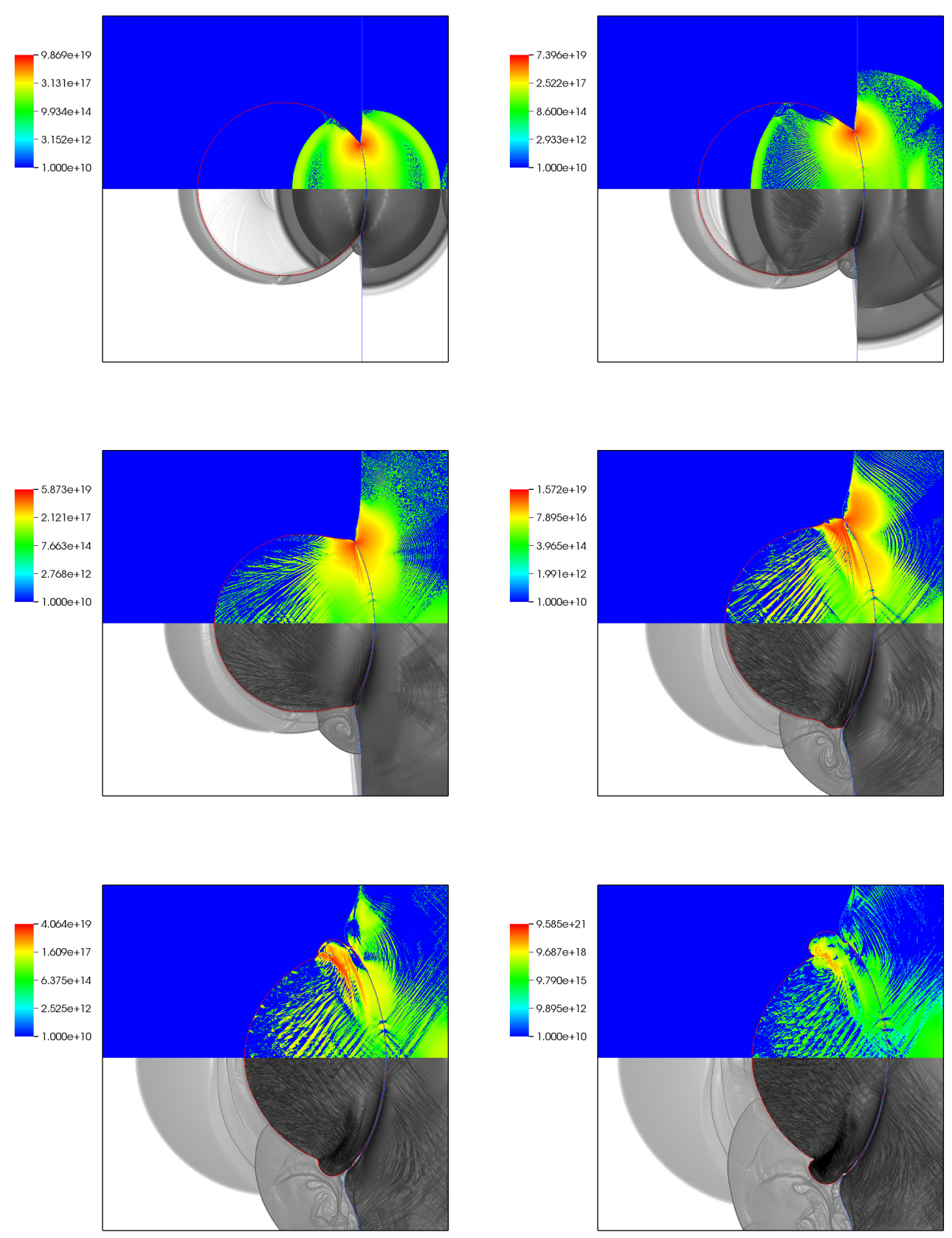

Fig. 10. TC3: Impact of copper on copper. Schlieren image and von Mises criteria at $t=120 \mu \mathrm{s}, 180 \mu \mathrm{s}, 328 \mu \mathrm{s}, 540 \mu \mathrm{s}, 760 \mu \mathrm{s}$ et $960 \mu \mathrm{s}$ from left to right, top to bottom 


\section{$5.33 D$ simulation}

In TC4, an iron sphere impacts an aluminium plate immersed in air. The initial configuration is presented in Fig 11 and the physical parameters are in Tab 6 . The computational domain is $[-0.3,0.7] \times[-0.4,0.4] \times[-0.4,0.4] \mathrm{m}^{3}$. The initial velocity of the projectile is $1000 \mathrm{~m} . \mathrm{s}^{-1}$. The computation is performed on a $500 \times 400 \times 400$ mesh with 216 processors. Homogeneous Neumann conditions are imposed on the left and right borders and cantilever on the others.

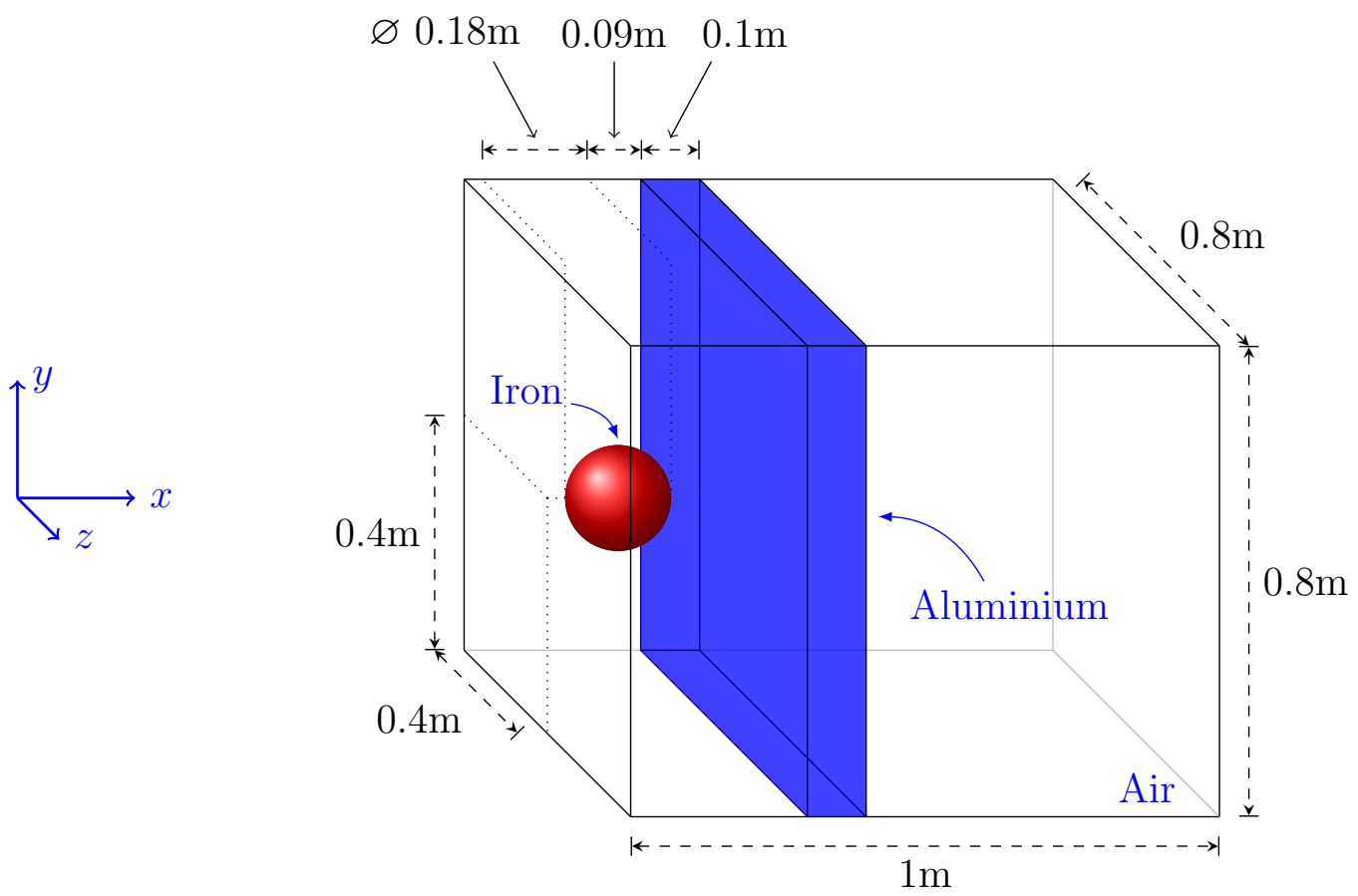

Fig. 11. Sketch of the initial configuration for TC4

\begin{tabular}{|l|c|c|c|c|c|c|}
\hline Media & $\rho\left(\mathrm{kg} . \mathrm{m}^{-3}\right)$ & $\gamma$ & $p_{\infty}(\mathrm{GPa})$ & $\chi(\mathrm{GPa})$ & $\sigma_{y}(\mathrm{GPa})$ & $\tau_{0}(\mathrm{~s})$ \\
\hline Air & 1 & 1.4 & 0 & 0 & - & - \\
\hline Iron & 7860 & 3.9 & 43.6 & 82 & 0.2 & $2.10^{-4}$ \\
\hline Aluminium & 2712 & 3.5 & 32 & 26 & 0.06 & $1.10^{-6}$ \\
\hline
\end{tabular}

Table 6

Parameters of materials for TC4

Fig 12 shows the material interfaces and the Schlieren results on the vertical symmetry plane. As in the $2 \mathrm{D}$ case, the projectile impacts the plates generating 
an elastic wave followed by a plastic wave. The waves are transmitted in the air at the rear of the plate and a jet of air emerges at the locations where the objects collide. The projectile perforates the aluminium plate which is strongly stretched. The breaking of the plate at final time is due to the level set function resolution and not to damage model.
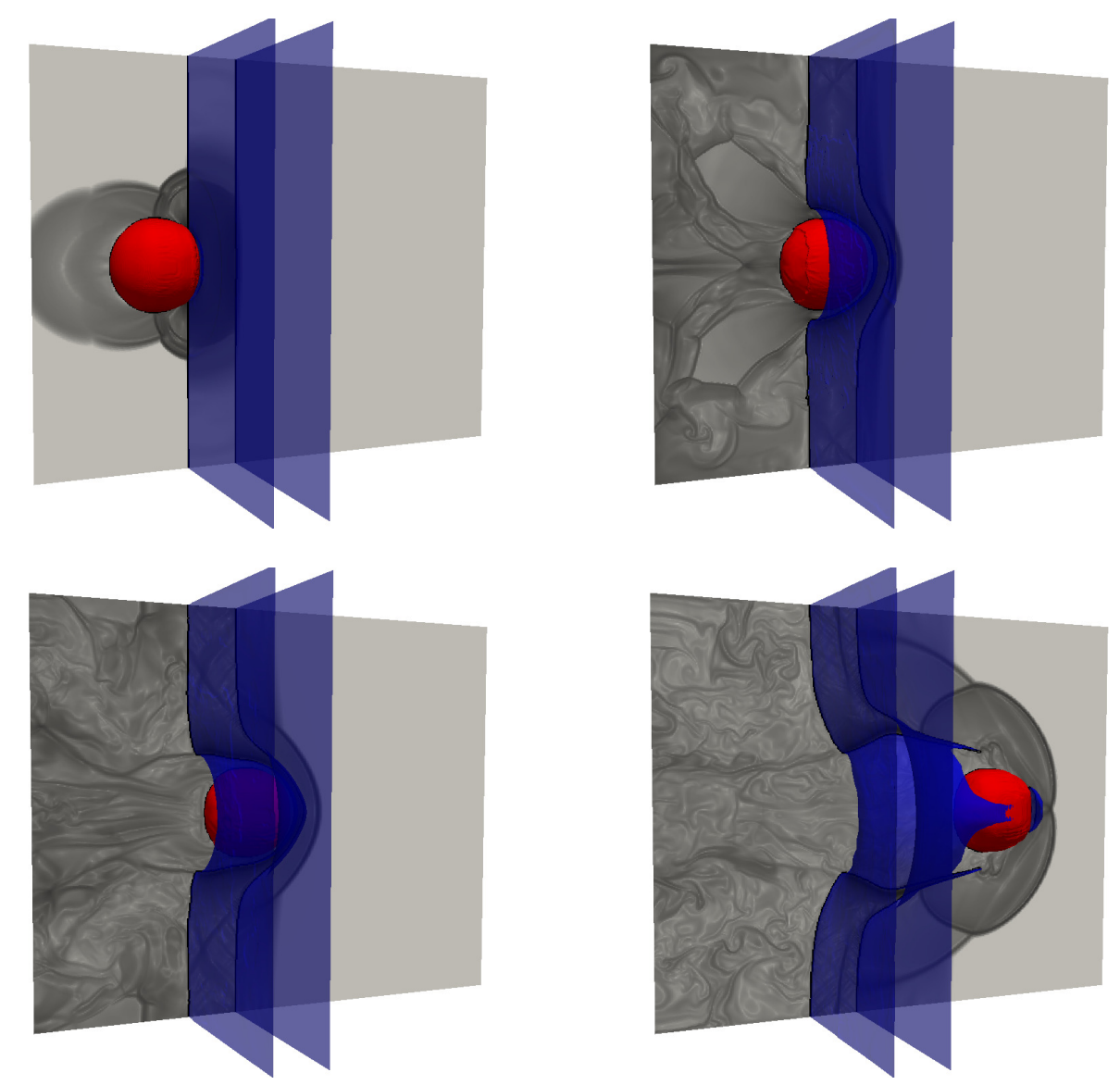

Fig. 12. TC4: Impact of Iron on Aluminium. Interfaces and Schlieren image at $t=110 \mu \mathrm{s}, 227 \mu \mathrm{s}, 337 \mu \mathrm{s}$ and $675 \mu \mathrm{s}$ from left to right, top to bottom

\section{Conclusions and Future Work}

We have presented a three-dimensional multimaterial model that describes the dynamics of the interaction between gas, liquids and elasto-plastic solids. We were explicitly able to show that the plasticity model for the neohookean hyperlastic constitutive law decreases the deviatoric norm. The numerical scheme is based on an approximate Riemann solver that is able to model multimate- 
rial interfaces and extends to plasticity the scheme presented in $[11,5]$. For hyperelastic-plastic compressible materials existing methods either rely on the definition of ghost materials or on mixture models and diffuse interfaces. In this paper we have proposed a simple, stable and non-oscillatory scheme for hyperelastic-plastic multimaterials that avoids the definition of a ghost medium or mixture models. Compared to such numerical models, this scheme is simpler as it does not require the storage of any additional variables or equations of state. This is a significant advantage for parallel schemes since communication overhead and algorithmic complexity are significantly reduced at the grid partitioning interfaces. Several stiff test cases of elasto-plastic impacts have been simulated thanks to this approach proving the robustness of this numerical model.

The explicit solver proposed is constrained by the time scale of the elastic waves and by the plasticity relaxation time. For problems such as impacts or in general fast-dynamical processes, this is the relevant scale of the physical phenomenon. For physical phenomena that take place on the time scale determined by the material velocity, the stability condition represents a significant limitation of these approches. Present developments are dedicated to this issue.

\section{Aknowledgements}

The simulations presented in this paper were carried out using the PLAFRIM experimental parallel testbed, being developed under the Inria PlaFRIM development action with support from LABRI and IMB and other entities: Conseil Régional d'Aquitaine, FeDER, Université de Bordeaux and CNRS (see https://plafrim.bordeaux.inria.fr/). Alexia de Brauer was supported by a grant from the DGA (Délégation Générale de l'Armement, Ministère de la Défense). 


\section{References}

[1] P.T. Barton, R. Deiterding, D. Meiron, and D. Pullin. Eulerian adaptative finite difference method for high-velocity impact and penetration problems. Journal of Computational Physics, 240:76-99, 2013. 2

[2] P.T. Barton, D. Drikakis, E. Romenski, and V.A. Titarev. Exact and approximate solutions of Riemann problems in non-linear elasticity. Journal of Computational Physics, 228(18):7046-7068, 2009. 2

[3] G.H. Cottet, E. Maitre, and T. Milcent. Eulerian formulation and level set models for incompressible fluid-structure interaction. ESAIM: Mathematical Modelling and Numerical Analysis, 42:471-492, 2008. 3

[4] S. Davis. Simplified second-order Godunov-type methods. SIAM Journal on Scientific and Statistical Computing, 9(3):445-473, 1988. 13

[5] A. de Brauer, A. Iollo, and T. Milcent. A Cartesian scheme for compressible multimaterial models in 3d. Journal of Computational Physics, 313:121-143, 2016. 2, 3, 4, 5, 12, 28

[6] N. Favrie and S. L. Gavrilyuk. Diffuse interface model for compressible fluid - Compressible elastic-plastic solid interaction. Journal of Computational Physics, 231(7):2695-2723, 2012. 2

[7] N. Favrie and S.L. Gavrilyuk. Dynamics of shock waves in elastic-plastic solids. ESAIM: Proceedings, 33:50-67, 2011. 11

[8] N. Favrie, S.L. Gavrilyuk, and R. Saurel. Solid-fluid diffuse interface model in cases of extreme deformations. Journal of Computational Physics, 228(16):6037-6077, 2009. 3, 4

[9] S.L. Gavrilyuk, N. Favrie, and R. Saurel. Modelling wave dynamics of compressible elastic materials. Journal of Computational Physics, 227(5):29412969, 2008. 2,4

[10] S.K. Godunov. Elements of continuum mechanics. Nauka Moscow, 1978. 2, 3

[11] Y. Gorsse, A. Iollo, T. Milcent, and H. Telib. A simple cartesian scheme for compressible multimaterials. Journal of Computational Physics, 272:772-798, 2014. $2,12,28$ 
[12] D.J. Hill, D. Pullin, M. Ortiz, and D. Meiron. An Eulerian hybrid WENO centered-difference solver for elasticplastic solids. Journal of Computational Physics, 229(24):9053-9072, 2010. 2

[13] A. Iollo, T. Milcent, and H. Telib. A sharp contact discontinuity scheme for multimaterial models. In Finite Volumes for Complex Applications VI, Problems \& Perspectives, volume 4 of Springer Proceedings in Mathematics, pages 581-588. Springer Berlin Heidelberg, 2011. 2, 12

[14] G.S. Jiang and C.W. Shu. Efficient implementation of Weighted ENO schemes. Journal of Computational Physics, 126(1):202-228, 1996. 14

[15] G. Kluth and B. Despres. Discretization of hyperelasticity on unstructured mesh with a cell-centered Lagrangian scheme. Journal of Computational Physics, 229(24):9092-9118, 2010. 2

[16] E.H. Lee and D.T. Liu. Finite-strain elastic-plastic theory with application to plane-wave analysis. Journal of Applied Physics, 38(1):19-27, 1967. 2, 6

[17] P.-H. Maire, R. Abgrall, J. Breil, R. Loubère, and B. Rebourcet. A nominally second-order cell-centered Lagrangian scheme for simulating elastic-plastic flows on two-dimensional unstructured grids. Journal of Computational Physics, 235(C):626-665, 2013. 2

[18] G.H. Miller and P. Colella. A high-order eulerian godunov method for elasticplastic flow in solids. Journal of Computational Physics, 167(1):131-176, 2001. 2,7

[19] G.H. Miller and P. Colella. A conservative three-dimensional eulerian method for coupled solid-fluid shock capturing. Journal of Computational Physics, 183(1):26-82, 2002. 2,3

[20] P.M. Naghdi. A critical review of the state of finite plasticity. Zeitschrift für angewandte Mathematik und Physik ZAMP, 41(3):315-394, 1990. 2

[21] S. Ndanou, N. Favrie, and S. Gavrilyuk. Multi-solid and multi-fluid diffuse interface model: Applications to dynamic fracture and fragmentation. Journal of Computational Physics, 295:523-555, 2015. 2, 15, 17

[22] A López Ortega, M Lombardini, D I Pullin, and D I Meiron. Numerical simulation of elasticplastic solid mechanics using an Eulerian stretch tensor approach and HLLD Riemann solver. Journal of Computational Physics, 257(PA):414-441, 2014. 2 
[23] B.J. Plohr and D.H. Sharp. A conservative eulerian formulation of the equations for elastic flow. Advances in Applied Mathematics, 9:481-499, 1988. 3

[24] B.J. Plohr and D.H. Sharp. A conservative formulation for plasticity. Advances in Applied Mathematics, 13:462-493, 1992. 3

[25] C.D. Sijoy and S. Chaturvedi. An Eulerian multi-material scheme for elasticplastic impact and penetration problems involving large material deformations. European Journal of Mechanics - B/Fluids, 53:85-100, 2015. 21

[26] E.F. Toro, M. Spruce, and W. Speares. Restoration of the contact surface in the HLL-Riemann solver. Shock Waves, 4:25-34, 1994. 12 